\title{
A mosaic mutation of phosphate-regulating gene with homologies to endopeptidases on the $X$ chromosome (PHEX) in X-linked hypophosphatemic rickets with mild bone phenotypes
}

\author{
Shoko Asano ${ }^{1)}$, Saori Sako ${ }^{1)}$, Yuka Funasaki ${ }^{1)}$, Yumie Takeshita ${ }^{1)}$, Yo Niida ${ }^{2)}$ and Toshinari Takamura' ${ }^{1)}$ \\ 1) Department of Endocrinology and Metabolism, Kanazawa University Graduate School of Medical Sciences, Ishikawa 920-8640, \\ Japan \\ 2) Division of Genomic Medicine, Department of Advanced Medicine, Medical Research Institute, Kanazawa Medical University, \\ Ishikawa 920-0293, Japan
}

\begin{abstract}
X-linked hypophosphatemic rickets $(\mathrm{XLH})$ is primarily characterized by renal phosphate wasting with hypophosphatemia, short stature, and bone deformity of the leg. Here we present a male case of XLH with relatively mild bone deformity caused by a mosaic mutation of the phosphate-regulating gene with homologies to endopeptidases on the $\mathrm{X}$ chromosome (PHEX). Polymerase chain reaction (PCR) direct sequencing revealed a novel in-frame deletion, NM-000444.6:c.671-685del p.Gln224-Ser228del, at exon 6 in PHEX as a mosaic pattern. This mutation was not found in any database and may result in a significant change in higher-order protein structure and function. TA cloning of the PCR product and clone sequencing estimated the mutation allele frequency at $21 \%$. Literature review of the previously reported three cases with novel mosaic mutations in PHEX, together with the present case, suggests that the rates of the mutation allele correlate with phenotype severity to some extent. We initially treated him with nutritional vitamin D supplements and phosphate salts. However, to avoid the development of secondary/tertiary hyperparathyroidism, we had switched nutritional to active vitamin D supplementation with reduced phosphorus salts. The present report contributes to understanding the relationship between the mosaic rate, in addition to the mutation locus, of the PHEX gene, and clinical features of XLH.
\end{abstract}

Key words: X-linked hypophosphatemic rickets, Phosphate-regulating gene with homologies to endopeptidases on the $\mathrm{X}$ chromosome, Rickets, Mosaicism

\section{X-LINKED HYPOPHOSPHATEMIC RICKETS}

(XLH) is the most common form of hereditary rickets, with a prevalence of $1 / 20,000$ [1]. Affected individuals have lifelong hypophosphatemia that results from lossof-function mutations of the gene encoding phosphateregulating gene with homologies to endopeptidases on the $\mathrm{X}$ chromosome (PHEX) that cause excess circulating levels of fibroblast growth factor 23 (FGF23). FGF23, which comprises 251 amino acids, is a hormone produced mainly by osteoblasts and osteocytes and regulates phosphate homeostasis and vitamin D metabolism via a specific FGF receptor- $\alpha$-klotho-complex in tubular

Submitted Dec. 22, 2020; Accepted Apr. 1, 2021 as EJ20-0809 Released online in J-STAGE as advance publication Apr. 28, 2021 Correspondence to: Toshinari Takamura, MD, PhD, Department of Endocrinology and Metabolism, Kanazawa University Graduate School of Medical Sciences, 13-1 Takara-machi, Kanazawa, Ishikawa 920-8640, Japan.

E-mail: ttakamura@med.kanazawa-u.ac.jp kidney cells. Excess FGF23 impairs renal phosphate reabsorption and decreases the synthesis of the active metabolite of vitamin D, 1,25-dihydroxyvitamin D3 (1,25-[OH]2D3), leading to hypophosphatemia [2]. As a result, extraskeletal ossification, osteoarthritis, joint mobility limitations, and occasionally spinal cord compression may develop.

The human PHEX gene comprises 22 exons that encode a 749-amino-acid protein and is strongly expressed in osteoblasts and osteocytes [3]. Since the PHEX gene mutation was first identified in 1995, at least 407 mutations, including nonsense mutations, have been registered in the Human Gene Mutation Database (http://www.hgmd.cf.ac.uk/ac/index.php). However, how PHEX gene mutations increase FGF23 levels and the relationship between mutation locus, type of mutation, and heterogeneous clinical features of XLH remain unknown. 


\section{Case Report}

A 61-year-old Japanese man presented to our hospital for a detailed examination of his coxodynia. He was unmarried and had no children. He had short stature $(150 \mathrm{~cm})$, bow legs, and scoliosis. He had never experienced any low-trauma fracture and lacked a family history of short stature, pathological fractures, and any congenital diseases. He was born at full term by normal vaginal delivery. He had been short in stature since his childhood. He was diagnosed with rickets at 5 years of age and received treatment with active vitamin $\mathrm{D}$ by age 10. During his growth spurt, he grew $30 \mathrm{~cm}$ to reach $150 \mathrm{~cm}$ tall. He had suffered from coxodynia since he was in his 40s. During the same period, he developed multiple caries.

The patient's laboratory test data on admission (Table 1) revealed hypophosphatemia, slightly elevated bonespecific alkaline phosphatase, reduced renal function, reduced 25-hydroxyvitamin D3 (25-[OH]D3), elevated parathyroid hormone (PTH) level, reduced renal tubular phosphorus reabsorption, and elevated FGF23 (assayed using the FGF-23 ELISA Kit, Kainos Laboratories, Tokyo). X-ray examinations revealed curvature of the spine (Fig. 1A), Looser's zones in the femurs, and ectopic ossification in the pelvis (Fig. 1B). The space of both hip joints was narrow with irregular margins (Fig. 1B). Based on the findings of bone changes, hypophosphatemia, and elevated FGF23 levels, FGF23related hypophosphatemic rickets was suspected.

Because the patient's bone pain symptom was mild and because his $25-(\mathrm{OH}) \mathrm{D} 3$ level was as low as $10.6 \mathrm{ng} / \mathrm{mL}$, we initially treated him with nutritional vitamin D supplements ( $25 \mu \mathrm{g}$ /day) and phosphate salts (600 mg/day). Nutritional vitamin D supplementation normalized his 25-(OH)D3 and 1,25-(OH)2D3 levels from 10.6 to $34.2 \mathrm{ng} / \mathrm{mL}(>20 \mathrm{ng} / \mathrm{mL})$ and from 42.3 to $69.4 \mathrm{pg} / \mathrm{mL}$ (20-60 pg/mL), respectively (Table 1). However, considering that FGF23 suppresses the activity of $1 \alpha$-hydroxylase, the conversion of $25-(\mathrm{OH}) \mathrm{D} 3$ to $1,25-$ $(\mathrm{OH}) 2 \mathrm{D} 3$ may be relatively insufficient. Indeed, PTH levels were elevated from 102 to $239 \mathrm{pg} / \mathrm{mL}$ during the course. To avoid the development of secondary/tertiary hyperparathyroidism, we thereafter had treated him with

Table 1 Laboratory data of the patient on admission

\begin{tabular}{|c|c|c|c|c|}
\hline & Before & $\begin{array}{l}\text { After treatment with nutritional } \\
\text { vitamin D and phosphate salts }\end{array}$ & $\begin{array}{l}\text { After treatment with active } \\
\text { vitamin } D \text { and phosphate salts }\end{array}$ & Reference range \\
\hline Phosphate (mg/dL) & 1.8 & 3.2 & 2.5 & $2.3-4.5$ \\
\hline Calcium (mg/dL) & 9.3 & 8.5 & 8.6 & $8.2-10.2$ \\
\hline Alkaline phosphatase (IU/L) & 373 & 417 & 450 & $110-330$ \\
\hline $\mathrm{BAP}(\mu \mathrm{g} / \mathrm{L})$ & 24.7 & 24.8 & & $3.7-20.9$ \\
\hline AST (IU/L) & 20 & 18 & 15 & $12-31$ \\
\hline $\operatorname{ALT}(\mathrm{IU} / \mathrm{L})$ & 24 & 19 & 14 & $8-40$ \\
\hline$\gamma$-GTP (IU/L) & 29 & 25 & 18 & $11-73$ \\
\hline Albmin (g/dL) & 4.5 & 3.9 & 4 & $3.8-5.3$ \\
\hline $\mathrm{UN}(\mathrm{mg} / \mathrm{dL})$ & 17 & 18 & 22 & $8-22$ \\
\hline Creatinin $(\mathrm{mg} / \mathrm{dL})$ & 1.21 & 1.38 & 1.3 & $0.6-1.1$ \\
\hline estimared GFR $\mathrm{cr}\left(\mathrm{mL} / \mathrm{min}\right.$ per $\left.1.73 \mathrm{~m}^{2}\right)$ & 48.4 & 39.16 & 44.13 & $\geqq 90$ \\
\hline cystatin $\mathrm{C}(\mathrm{mg} / \mathrm{L})$ & & 1.47 & & \\
\hline estimared GFR cys (mL/min per $\left.1.73 \mathrm{~m}^{2}\right)$ & & 46.3 & & \\
\hline 25-(OH)D3 (ng/mL) & 10.6 & 34.2 & & $>20$ \\
\hline 1,25-(OH)2D3 (pg/mL) & 42.3 & 69.4 & & $20-60$ \\
\hline PTH (pg/mL) & 102.2 & 248 & 168 & $10.3-65.9$ \\
\hline FGF23 (pg/mL) & 104 & 239 & & $<30$ \\
\hline $\mathrm{TmP} / \mathrm{GFR}(\mathrm{mg} / \mathrm{mL})$ & 1.2 & 1.42 & 1.17 & $2.3-4.3$ \\
\hline TRP (\%) & 66.72 & 44.46 & 46.7 & $81-90$ \\
\hline
\end{tabular}

BAP, bone specific alkaline phosphatase; GFR, glomerular filtration rate; UN, urea nitrogen; 25-(OH)D3, 25-hydroxyvitamin D3; 1,25$(\mathrm{OH}) \mathrm{D} 3$, 1,25-dihydroxyvitamin D3; PTH, parathyroid hormone; FGF23, fibroblast growth factor 23; TmP, tubular maximum phosphate reabsorption; TRP, tubular reabsorption of phosphate. 
A

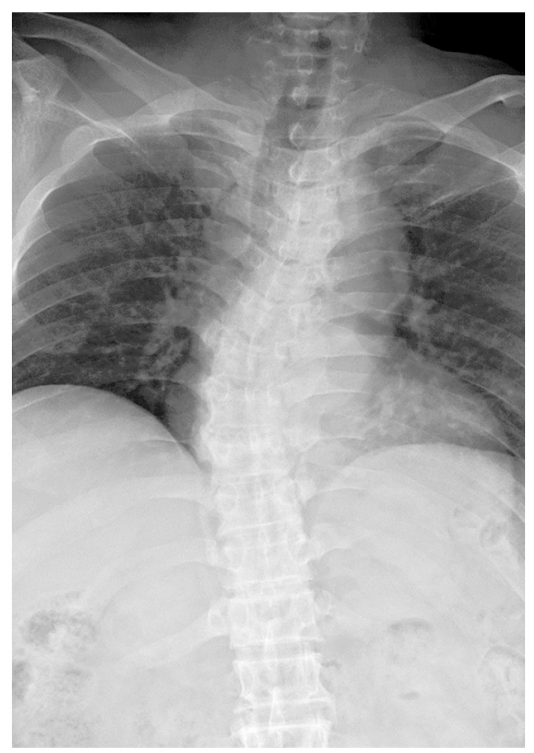

B

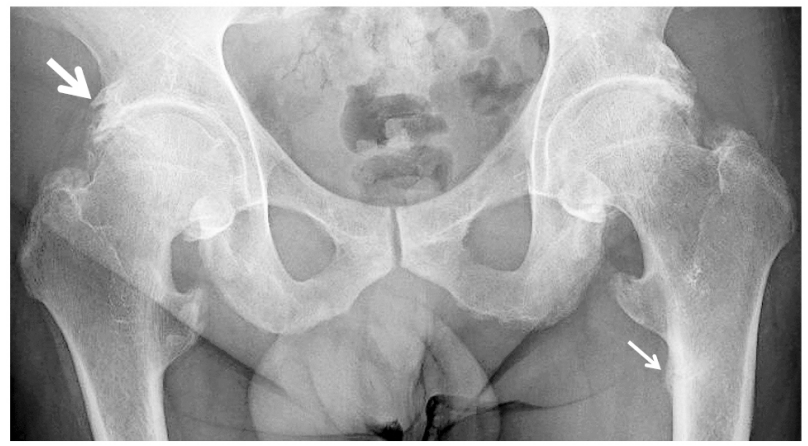

Fig. 1 X-ray examinations of the spine (A) and pelvis/femoral heads (B)

A: Spine curvature.

B: Looser's zones in the femurs (thin arrow). Ectopic ossification (thick arrow) in the pelvis. The space of both hip joints was narrow with irregular margins.

active vitamin D and phosphate salts, which reduced his PTH level to $168 \mathrm{pg} / \mathrm{mL}$ after 3 months (Table 1).

\section{Materials and Methods}

Genomic DNA was extracted from whole blood using a rapid extraction method [4]. The mutation was screened by CHIPS (CEL nuclease mediated heteroduplex incision with polyacrylamide gel electrophoresis and silver staining) assay, a highly sensitive enzyme mismatch cleavage assay described elsewhere [5, 6]. Subsequently, the mutation was confirmed by Sanger sequencing using the BigDye Terminator v3.1 Cycle Sequencing Kit on a 3100XL Genetic Analyzer (Thermo Fisher Scientific). PCR primers used in the CHIPS and direct sequencing were shown in Table 2. To distinguish the shorter fragment of the mutation allele from the wild- type allele, we set PCR primers as forward primer PHEX-6F: 5'-AATATGGCTGGGATGCAGAC-3' and reverse primer PHEX-6AS: 5'-GAGTAAAACTTACAG ACTTGGCTTC-3' as shown in Fig. 2A. TA cloning of this PCR product was performed with pT7 Blue T-vector (Novagen). The mutation allele frequency was estimated by colony direct PCR and $12 \%$ polyacrylamide gel electrophoresis for 100 colonies.

Written informed consent was obtained from the patient after he received courteous genetic counseling and an explanation of the genetic testing, as approved by the institutional review board (No. G111).

\section{Genetic Analyses}

We performed a genetic analysis for the candidate genes involved in FGF23-related hypophosphatemic rickets. We identified a shorter PCR fragment derived from the PHEX gene at exon 6 (Fig. 2A) in the patient. Sanger sequencing revealed a PHEX in-frame deletion NM_000444.6:c.671_685del p.Gln224_Ser228del as a mosaic pattern. Clone PCR of 100 colonies estimated the mutation allele frequency $21 \%$ (Fig. 2B), suggesting the mosaic mutation. This mutation was not found in any database, including the Genome Aggregation Database (http://gnomad.broadinstitute.org), the Exome Aggregation Consortium (http://exac.broadinstitute.org), and the Human Gene Mutation Database, suggesting that this mutation is a novel and not a normal variant.

The deleted region of the PHEX gene (Gln224 Ser228) in this patient is completely conserved among mammalian species, for example, human, mouse, and bat. Using the software tool Sequence to Function Annotation Server (http://citeseerx.ist.psu.edu), we could see that in the high-order structure of the DNA around the mutation, the deletion exists between the $\beta$-sheet structure and $\alpha$-helix structure. The deletion causes the shortage of the $\beta$-sheet structure and makes the protein structure unstable. Using the software tool PROVEAN (http://provean.jcvi.org/index.php), we also predicted the impact of this novel mutation on protein structure by in silico analyses. These in silico prediction engines resulted in a score of -21.52 , which suggests deleterious damage in PHEX function. These results suggest that this region is critical for the function of the protein and is causal for XLH. From these results, we diagnosed the patient's illness as XLH caused by a mosaic novel PHEX mutation.

\section{Discussion}

We report a novel $P H E X$ in-frame deletion mutation as a mosaic pattern in a sporadic male case of XLH with 
Table 2 PHEX each exon PCR primer for CHIPS and direct sequencing

\begin{tabular}{|c|c|c|c|}
\hline Exon* & Primer Name & Primer Seq & Product size (bp) \\
\hline \multirow{2}{*}{1} & PHEX-1F & 5'-GAGCAAGAAAGCCTTGGATG-3' & \multirow{2}{*}{334} \\
\hline & PHEX-1R & 5'-ACCTATGAACGCAGGCAAAC-3' & \\
\hline \multirow{2}{*}{2} & PHEX-2F & 5'-TGTTTCCGAGGGTGGTTTAC-3' & \multirow{2}{*}{266} \\
\hline & PHEX-2R & 5'-CAAAATTGCATTTCGTACTTGG-3' & \\
\hline \multirow{2}{*}{3} & PHEX-3F & 5'-GGGGAAATATAAATGCCAAGG-3' & \multirow{2}{*}{345} \\
\hline & PHEX-3R & 5'-TGTTTTTCCTGTCAAATATTGCTC-3' & \\
\hline \multirow{2}{*}{4} & PHEX-4F & 5'-TTTCTGGAGGTTGGAATTGTG-3' & \multirow{2}{*}{324} \\
\hline & PHEX-4R & 5'-CTTTCACAATCATTCCTTTAACACC-3' & \\
\hline \multirow{2}{*}{5} & PHEX-5F & 5'-CTAGTGTGCTGATCCAGTTTGC-3' & \multirow{2}{*}{371} \\
\hline & PHEX-5R & 5'-GGCAGCATGAGTCTCTTTCC-3' & \\
\hline \multirow{2}{*}{6} & PHEX-6F & 5'-AATATGGCTGGGATGCAGAC-3' & \multirow{2}{*}{293} \\
\hline & PHEX-6R & 5'-CCTGCATTGGGAATATGGTC-3' & \\
\hline \multirow{2}{*}{7} & PHEX-7F & 5'-TTCTGCTCTTCCATGTCTCTCA-3' & \multirow{2}{*}{298} \\
\hline & PHEX-7R & 5'-ATTTTCATTCAGGATATACTGCCTTT-3' & \\
\hline \multirow{2}{*}{8} & PHEX-8F & 5'-CAGATGTTTTGGCACATGTAGG-3' & \multirow{2}{*}{278} \\
\hline & PHEX-8R & 5'-ACCAAGACTGAGAGCAGGTTTTAC-3' & \\
\hline \multirow{2}{*}{9} & PHEX-9F & 5'-GATTCTCTCATTCTGTTTTGTTCTCTC-3' & \multirow{2}{*}{260} \\
\hline & PHEX-9R & 5'-AAAGGATGTGAGAAGGGAAGC-3' & \\
\hline \multirow{2}{*}{10} & PHEX-10F & 5'-GGAGCTTTGCCAACTGTTTC-3' & \multirow{2}{*}{294} \\
\hline & PHEX-10R & 5'-CCCCTGTCTAATCCCTAAAGATG-3' & \\
\hline \multirow{2}{*}{11} & PHEX-11F & 5'-TTCAGCCATGGGTTTTATCC-3' & \multirow{2}{*}{282} \\
\hline & PHEX-11R & 5'-CTACCACCTGGAAGGCTGAC-3' & \\
\hline \multirow{2}{*}{12} & PHEX-12F & 5'-GCTTCTTGGCTTTGACGTTC-3' & \multirow{2}{*}{263} \\
\hline & PHEX-12R & 5'-GGGGTCATTCAGAGTCAACAG-3' & \\
\hline \multirow{2}{*}{13} & PHEX-13F & 5'-GAAGGGCGCATTTCTACATC-3' & 273 \\
\hline & PHEX-13R & 5'-TTGGTGTTTTCTGGCATCAC-3' & 213 \\
\hline 14 & PHEX-14F & 5'-TTGCTCCTTCCTATGCTGAAG-3' & 300 \\
\hline 17 & PHEX-14R & 5'-AACTGGCAAGCCAGCTACTC-3' & 300 \\
\hline 15 & PHEX-15F & 5'-GTCCAACATCCCCATTGTTC-3' & 297 \\
\hline 10 & PHEX-15R & 5'-AGCATACCCTGCCACTTTTG-3' & 2 \\
\hline 16 & PHEX-16F & 5'-GAGGAGTGCCTTTCAGATGG-3' & 276 \\
\hline 10 & PHEX-16R & 5'-CCATGGCTTCTTTCTGCTG-3' & 210 \\
\hline 17 & PHEX-17F & 5'-CAGTTTATCTTGGCTTTCCATTG-3' & 264 \\
\hline 17 & PHEX-17R & 5'-AAGCTATGATGCTGCTGCTATG-3' & 204 \\
\hline 18 & PHEX-18F & 5'-CTTGTCGAGGTGAGGGAAAG-3' & 287 \\
\hline 10 & PHEX-18R & 5'-TGGTTTAAAGCAAGCTAATGTCC-3' & 201 \\
\hline 19 & PHEX-19F & 5'-GCCTACCTCTGATTTATTGAATGTG-3' & 297 \\
\hline & PHEX-19R & 5'-AAGAACATGGCTATGGTATGAATTG-3' & \\
\hline 20 & PHEX-20F & 5'-TTTGCTATTCCTGTGCTAGCTG-3' & 240 \\
\hline 20 & PHEX-20R & 5'-GGGAGCAAACTCAAGTCCTG-3' & 240 \\
\hline 21 & PHEX-21F & 5'-ATTTGGAGCAGTTAAAACAGCAG-3' & 239 \\
\hline 21 & PHEX-21R & 5'-TCTGGTAGAGCCCTTGGATG-3' & 239 \\
\hline 22 & PHEX-22F & 5'-AGAATGCCAACCTTCTTTCTAGC-3' & 299 \\
\hline 22 & PHEX-22R & 5'-TCTCCAGGCCTAAAGCAATG-3' & 29 \\
\hline
\end{tabular}

* Exon number is according to Genbank NM_000444.5, transcript variant 1 (https://www.ncbi.nlm. nih.gov/nuccore/NM_000444.5) 
A

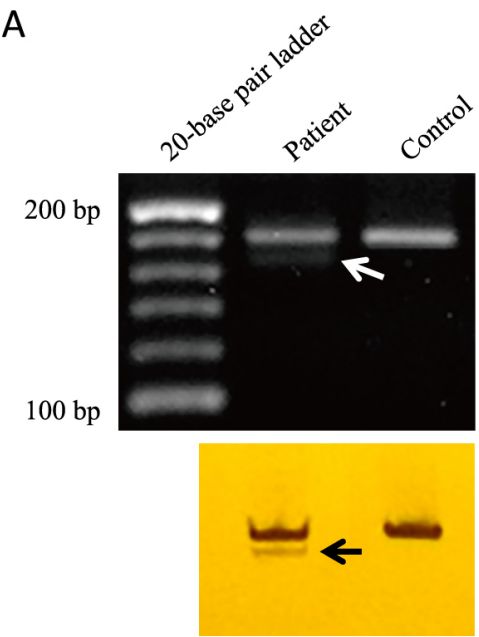

B

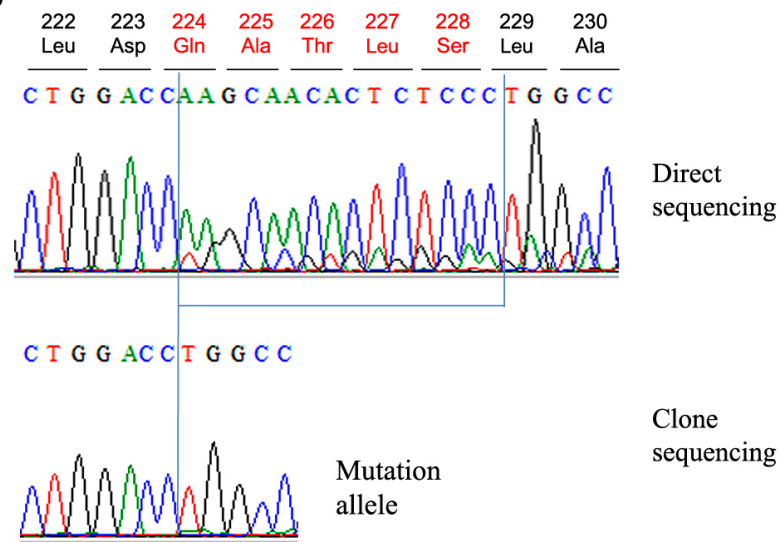

Fig. 2 Mosaic mutation in the PHEX gene

A: The $3 \%$ agarose (top) and $12 \%$ polyacrylamide gel electrophoresis identified a shorter fragment derived from the PHEX gene at exon 6 (arrow).

B: PCR-direct Sanger sequencing detected low mosaic peaks. Cloning the PCR product and clone sequencing revealed the PHEX in-frame deletion mutation. Clone PCR of 100 colonies revealed 79 wild-type and 21 mutant alleles (allele frequency $21 \%$ ).

relatively mild bone deformity. We found it difficult to diagnose this patient as XLH because of his relatively mild symptoms and absence of family history. Of note, frequency of sporadic XLH are as high as 30\% [7], 53\% [8], and 54\% [9]. PHEX mutation frequencies are less common in sporadic cases $(28 \%-73 \%)$ than familial cases (52\%-91\%) with XLH [8].

Some studies have reported genotype-phenotype correlations in XLH; however, there has been no definite relationship found between disease severity and type of mutation $[7,10]$. The mosaic mutation in PHEX appears to be rare. Indeed, there have been only three cases with novel mosaic mutations in PHEX reported in males [11-13]. Table 3 summarizes the clinical features of these cases. Among these cases, the Chinese boy [11] and the Japanese man [12] with a higher mutation rate seem to present relatively severer phenotypes. On the other hand, the Korean man [13] and the present patient with a lower mutation rate present a relatively milder phenotype. This trend in findings suggests that, in these cases, the rates of the mutation allele correlate with phenotype severity to some extent. Therefore, in patients with mild phenotypes, genetic analyses are strongly recommended to determine the mosaic somatic mutation of PHEX. However, we must keep in mind that mutation rates may vary among organs.

In the XLH subjects without impaired kidney function, serum FGF23 level negatively correlates with serum phosphate. However, FGF23 increases exponentially as eGFR declines [14]. Also, in the present patient, moderately impaired renal function is attributable to his elevated FGF23 levels. In this setting, elevated FGF23 levels are no longer consistent with his mild symptoms and the mosaic mutation of PHEX. Unfortunately, besides the present case, FGF23 levels were not reported in other cases with the PHEX mosaic mutation (Table 3). Accumulating similar cases will clarify the regulatory mechanisms of FGF23 production independently of the PHEX function as well as the role FGF23 plays in the disease severity of XLH.

Unfortunately, he had initially been treated with nutritional vitamin D supplements and phosphate salts. The majority of patients with XLH (83.3\%) were reported to have evidence of secondary hyperparathyroidism [15]. Transient increase in serum phosphorus after phosphate doses is expected to stimulate PTH production due to unidentified mechanism in XLH patients [16]. Indeed, prolonged use of high-dose phosphate therapy has been identified as a risk factor for PTH elevation [17]. Active vitamin D supplementation reduces elevated PTH levels. Of note, FGF23 suppresses 25-hydroxyvitamin D-1 $\alpha$ hydroxylase and activates 25-hydroxyvitamin D-24hydroxylase in the kidney [2]. In patients with XLH whose FGF23 levels are elevated, the nutritional vitamin D supplementation insufficiently elevates 1,25-(OH)2D3 levels. In addition, serum 1,25-(OH)2D3 levels represent 1,25- $(\mathrm{OH}) 2 \mathrm{D} 3$ production in the renal tubule, which correlates with PTH levels, while actual action of 1,25$(\mathrm{OH}) 2 \mathrm{D} 3$ in the intestine is only associated with $1,25-$ $(\mathrm{OH}) 2 \mathrm{D} 3$ production in the intestine by local $1 \alpha-$ hydroxylase and irrelevant to serum 1,25-(OH)2D3 levels. Therefore, his elevated serum 1,25-(OH)2D3 level after nutritional vitamin D supplementation may be attributable to the elevated PTH levels and does not represent his actual local 1,25-(OH)2D3 activity in the intestine. The treatment with phosphate under insufficient active vitamin D supplementation may aggravate 
Table 3 Summary of the clinical features of previously reported cases of X-linked hypophosphatemic rickets with a mosaic mutation of PHEX

\begin{tabular}{|c|c|c|c|c|}
\hline & \multicolumn{4}{|c|}{ Case reference number } \\
\hline & 11 & 12 & 13 & Present case \\
\hline $\begin{array}{l}\text { Age (year)/Gender/ } \\
\text { Nationality }\end{array}$ & 4.5/M/China & 35/M /Japan & 26/M/Korea & 61/M/Japan \\
\hline Family history & - & Proband's daughter & - & - \\
\hline Bone phenotypes & $\begin{array}{l}\text { Caput quadratum } \\
\text { Bow legs }\end{array}$ & Bow legs & $\begin{array}{l}\text { Mild osteoarthritis in } \\
\text { both joint } \\
\text { Multiple old fractures }\end{array}$ & $\begin{array}{c}\text { Bow legs } \\
\text { Curvature of his spine }\end{array}$ \\
\hline Serum FGF23 level & N.D. & N.D. & N.D. & $104 \mathrm{pg} / \mathrm{mL}$ \\
\hline $\begin{array}{l}\text { Serum phosphate } \\
\text { level }\end{array}$ & $2.0 \mathrm{mg} / \mathrm{dL}$ & $1.7 \mathrm{mg} / \mathrm{dL}$ & $2.0 \mathrm{mg} / \mathrm{dL}$ & $1.8 \mathrm{mg} / \mathrm{dL}$ \\
\hline $\begin{array}{l}\text { Serum alkaline } \\
\text { phosphatase level }\end{array}$ & $616 \mathrm{IU} / \mathrm{L}$ & $215 \mathrm{IU} / \mathrm{L}$ & $99 \mathrm{IU} / \mathrm{L}$ & $373 \mathrm{IU} / \mathrm{L}$ \\
\hline Mutation in $P H E X^{*}$ & $\begin{array}{c}\text { c. } 1769-1 \mathrm{G}>\mathrm{A} \\
\text { p.Gly590GlufsTer28 }\end{array}$ & $\begin{array}{l}\text { c. } 1669 \mathrm{C}>\mathrm{T} \\
\text { p.Arg567Ter }\end{array}$ & $\begin{array}{l}\text { c. } 589 \mathrm{C}>\mathrm{T} \\
\text { p.Gln197Ter }\end{array}$ & $\begin{array}{c}\text { c.671_685del } \\
\text { p.Gln224_Ser228del }\end{array}$ \\
\hline Mosaic rate & $42 \%$ & $60 \%$ & $38 \%$ & $21 \%$ \\
\hline
\end{tabular}

N.D., not dertermined; * mRNA reference number is NM_000444.5

his secondary hyperparathyroidism. Therefore, we had switched nutritional to active vitamin D supplementation with reduced phosphorus salts to prevent the development of secondary/tertiary hyperparathyroidism according to the clinician's guide to XLH [16], resulting in a reduction in his PTH level.

Recently, burosumab, a human anti-FGF23 antibody, was developed as a potential treatment for XLH. In children with XLH, treatment with burosumab increased renal tubular phosphate reabsorption, serum phosphorus levels, linear growth, and physical function and reduced the pain and severity of rickets [18]. In adults with XLH, the treatment increased serum phosphorus levels and mobility and reduced pain and the severity of rickets [19]. Therefore, to improve patients' quality of life, early definite diagnosis is of great importance in XLH, although the indication for burosumab treatment in adults with XLH should be carefully discussed.

In summary, we found a novel PHEX in-frame deletion NM_000444.6:c.671_685del p.Gln224_Ser228del mutation as a mosaic pattern in a sporadic male case of XLH with relatively mild bone deformity. The mutation may result in a significant change in higher-order protein structure and function and might, through an increase in the production of FGF23, cause XLH. Mild symptoms of rickets may be associated with the PHEX mutation presented as a mosaic pattern. We believe the present report contributes to understanding the relationship between the mosaic rate, in addition to the mutation locus, of the PHEX gene, and clinical features of XLH.

\section{Disclosure}

None of the authors have any potential conflicts of interest associated with this research.

\section{Disclosure Statement}

The authors have nothing to disclose.

\section{References}

1. Endo I, Fukumoto S, Ozono K, Namba N, Inoue D, et al. (2015) Nationwide survey of fibroblast growth factor 23 (FGF23) - related hypophosphatemic diseases in Japan: prevalence, biochemical data and treatment. Endocr $J 62$ : 811-816.

2. Shimada T, Hasegawa H, Yamazaki Y, Muto T, Hino R, et al. (2004) FGF-23 is a potent regulator of vitamin D metabolism and phosphate homeostasis. J Bone Miner Res 19: 429-435.

3. Beck L, Soumounou Y, Martel J, Krishnamurthy G, Gauthier C, et al. (1997) Pex/PEX tissue distribution and evidence for a deletion in the 3 ' region of the Pex gene in X-linked hypophosphatemic mice. J Clin Invest 99: 12001209. 
4. Lahiri DK, Schnabel B (1993) DNA isolation by a rapid method from human blood samples: effects of $\mathrm{MgCl}_{2}$, EDTA, storage time, and temperature on DNA yield and quality. Biochem Genet 31: 321-328.

5. Niida Y, Kuroda M, Mitani Y, Okamura A, Yokoi A (2012) Applying and testing the conveniently optimized enzyme mismatch cleavage method to clinical DNA diagnosis. Mol Genet Metab 107: 580-585.

6. Tsuji T, Niida Y (2008) Development of a simple and highly sensitive mutation screening system by enzyme mismatch cleavage with optimized conditions for standard laboratories. Electrophoresis 29: 1473-1483.

7. Holm IA, Nelson AE, Robinson BG, Mason RS, Marsh DJ, et al. (2001) Mutational analysis and genotypephenotype correlation of the PHEX gene in X-linked hypophosphatemic rickets. J Clin Endocrinol Metab 86: 3889-3899.

8. Gaucher C, Walrant-Debray O, Nguyen TM, Esterle L, Garabédian M, et al. (2009) PHEX analysis in 118 pedigrees revels new genetic clues in hypophosphetamic rickets. Hum Genet 125: 401-411.

9. Basu D, Pettifor JM, Kromberg J (2004) X-linked hypophosphatemia in South Africa. S Afr Med J 94: 460-464.

10. Song HR, Park JW, Cho DY, Yang JH, Yoon HR, et al. (2007) PHEX gene mutations and genotype-phenotype analysis of Korean patients with hypophosphatemic rickets. J Korean Med Sci 22: 981-986.

11. Weng C, Chen J, Sun L, Zhou ZW, Feng X, et al. (2016) A de novo mosaic mutation of PHEX in a boy with hypophosphatemic ricket. J Human Genet 61: 223-227.

12. Goji K, Ozaki K, Sadewa AH, Nishio H, Matsuo M
(2006) Somatic and germline mosaicism for a mutation of the PHEX gene can lead to genetic transmission of Xlinked hypophosphatemic rickets that mimics an autosomal dominant trait. J Clin Endocrinol Metab 91: 365370.

13. Yang M, Kim J, Yong A, Jang J, Jeon TY, et al. (2018) A novel de novo mosaic mutation in PHEX in a Korean patient with hypophosphatemic rickets. Ann Pediatr Endocrinol Metab 23: 229-234.

14. Wolf M (2012) Update on fibroblast growth factor 23 in chronic kidney disease. Kidney Int 87: 737-747.

15. Delacey S, Liu Z, Broyles A, El-Azab SA, Guandique CF, et al. (2019) Hyperparathyroidism and parathyroidectomy in X-linked hypophosphatemia patients. Bone 127: 386392.

16. Carpenter TO, Imel EA, Holm IA, Jan de Beur SM, Insogna KL (2011) A clinician's guide to X-linked hypophosphatemia. J Bone Miner Res 26: 1381-1388.

17. Makitie O, Kooh SW, Sochett E (2003) Prolonged highdose phosphate treatment: a risk factor for tertiary hyperparathyroidism in X-linked hypophosphatemic rickets. Clin Endocrinol (Oxf) 58: 163-168.

18. Carpenter TO, Whyte MP, Imel EA, Boot AM, Högler W, et al. (2018) Burosumab therapy in children with X-linked hypophosphatemia. N Engl J Med 378: 1987-1998.

19. An Insogna KL, Briot K, Imel EA, Kamenický P, Ruppe $\mathrm{MD}$, et al. (2018) A randomized, double-blind, placebocontrolled, phase 3 trial evaluating the efficacy of burosumab, anti-FGF23 antibody, in adults with Xlinked hypophosphatemia: Week 24 primary analysis. $J$ Bone Miner Res 33: 1383-1393. 